Published in final edited form as:

Nature. ; 485(7400): 656-660. doi:10.1038/nature11095.

\title{
Inhibitory receptors bind Angptls and support blood stem cells and leukemia development
}

\author{
Junke Zheng ${ }^{1,3,7}$, Masato Umikawa ${ }^{1,4,7}$, Changhao Cui ${ }^{1,7}$, Jiyuan Li $^{1}$, Xiaoli Chen ${ }^{1}$, \\ Chaozheng Zhang ${ }^{1}$, HoangDinh Hyunh ${ }^{1}$, Xunlei Kang ${ }^{1}$, Robert Silvany ${ }^{1}$, Xuan Wan ${ }^{1}$, \\ Jingxiao $\mathrm{Ye}^{1}$, Alberto Puig Cantó ${ }^{2}$, Shu-Hsia Chen ${ }^{5}$, Huan-You Wang ${ }^{6}$, E. Sally Ward ${ }^{2}$, and \\ Cheng Cheng Zhang ${ }^{1,8}$ \\ ${ }^{1}$ Departments of Physiology and Developmental Biology, University of Texas Southwestern \\ Medical Center, Dallas, Texas 75390, USA \\ ${ }^{2}$ Department of Immunology, University of Texas Southwestern Medical Center, Dallas, Texas \\ 75390, USA
}

${ }^{3}$ Key Laboratory of Cell Differentiation and Apoptosis of Chinese Ministry of Education, Shanghai Jiao Tong University School of Medicine, Shanghai 200025, China

${ }^{4}$ Department of Medical Biochemistry, University of the Ryukyus, Okinawa 903-0215, Japan

${ }^{5}$ Department of Oncological Sciences, Mount Sinai School of Medicine, New York, NY 10029-6574, USA

${ }^{6}$ Department of Pathology, University of California San Diego, La Jolla, CA 92093, USA

\begin{abstract}
How the activities of adult stem cells and cancer cells are regulated by environmental cues through surface receptors is poorly understood. Angiopoietin-like proteins (Angptls), a family of seven secreted glycoproteins, are known to support the activity of hematopoietic stem cells (HSCs) in vitro and in vivo ${ }^{1-10}$. Angptls also play important roles in lipid metabolism, angiogenesis, and inflammation but were considered "orphan ligands" as no receptors were identified ${ }^{3,11,12}$. Here we report that the immune inhibitory receptors, human leukocyte immunoglobulin (Ig)-like receptor B2 (LILRB2) and its mouse ortholog paired Iglike receptor (PirB), are receptors for several Angptls. LILRB2 and PirB are expressed on human HSCs and mouse HSCs, respectively. Angptls bound to LILRB2 and to PirB and supported ex vivo expansion of HSCs. In the mouse MLL-AF9 and AML1-ETO9a transplantation acute myeloid leukemia (AML) models, a deficiency in intracellular signaling of PirB resulted in increased differentiation of leukemia cells, revealing that PirB
\end{abstract}

\footnotetext{
Users may view, print, copy, download and text and data- mine the content in such documents, for the purposes of academic research, subject always to the full Conditions of use: http://www.nature.com/authors/editorial_policies/license.html\#terms

${ }^{8}$ Corresponding Author: Cheng Cheng Zhang, Telephone (214) 645-6320, Fax (214) 648-1960, Alec.Zhang@UTSouthwestern.edu.

7 These authors contributed equally to the work

Author Contributions

J.Z., M.U., C.C., and C.C.Z.: study design, identification of receptors, binding, signaling, and functional assays, data analysis, and writing of manuscript. J.L., X.C., C.Z., H.H., X.K., R.S., X.W.: binding and signaling assays and data analysis. J.Y. and S.-H.C.: ligand binding assays. H.-Y.W.: AML characterization. A.P.C. and E.S.W.: SPR assay and data analysis.

DNA microarray data are available on NCBI GEO through accession number GSE36329. Reprints and permissions information is available at www.nature.com/reprints. The authors declare no competing financial interests.
} 
supports leukemia development. Our study indicates unexpected functional significance of classical immune inhibitory receptors in maintenance of stemness of normal adult stem cells and in support of cancer development.

We used multiple approaches, including expression cloning, to identify the receptor(s) for Angptls. Human LILRB2, when ectopically expressed on BAF3 cells, enabled the cells to specifically bind GST-Angpt15 as determined by flow cytometry (Fig. 1a). LILRB2 is a member of the immune inhibitory B type subfamily of LILR receptors ${ }^{13}$ and contains four Ig-domains and three immunoreceptor tyrosine-based inhibitory motifs. Using flow cytometry analysis, we further demonstrated that LILRB2-overexpressing 293T cells had enhanced binding to several Angptls, especially Angpt12 and GST-Angpt15 (Fig. 1b, Supplementary Fig. 1a-b). Angpt12 and GST-Angpt15 also bound to LILRB3- and LILRB5overexpressing cells, though with a lower affinity than to LILRB2-expressing cells (Supplementary Table 1). In addition, Angptl1 and Angptl7 bound to LAIR1 ${ }^{14}$ overexpressing 293T cells (Supplementary Table 1, Supplementary Fig. 2). Angptls did not bind to LILRAs, LILRB1, or LILRB4 (Supplementary Table 1).

Because Angpt12 and GST-Angpt15 bound to LILRB2-expressing cells better than did other Angptls, we further assessed the molecular interaction between Angpt12/Angpt15 and LILRB2. Co-transfection of Angpt12 or Angpt15 with LILRB2 extracellular domain (ECD) fused to human IgG-Fc (LILRB2-hFc) into 293T cells followed by immunoprecipitation (IP)/western blot revealed that both Angpt12 and Angptl5 interacted with the extracellular domain of LILRB2 but not that of Tie-2 (Fig. 1c, Supplementary Fig. 1c). The direct interactions between Angptls and LILRB2 were confirmed by in vitro co-IP using purified Angpt12-FLAG or GST-Angpt15 and LILRB2-hFc (Supplementary Fig. 1d) and by surface plasmon resonance (SPR) (Supplementary Fig. 3). A liquid-phase binding assay with ${ }^{125} \mathrm{I}$ labelled GST-Angpt15 demonstrated that the interaction between Angpt15 and cell surface LILRB2 was specific and saturable, with half maximal saturation of the interaction as $5.5 \pm$ $1.1 \mathrm{nM}$ (Figs. 1d-e). While untagged Angptls bind to LILRB2, the type or the position of tagging could affect the binding (Supplementary Table 2).

Because several Angptls support expansion of HSCs ${ }^{4-12}$, we sought to determine whether Angptls bound to LILRB2 or LAIR1 on primary human cord blood cells. Flow cytometry analysis showed that Angptls 1, 2, 5, and 7 all bound to LILRB2 ${ }^{+}$human cord blood cells; Angpt12 and GST-Angpt15 had higher affinities (Fig. 2a, Supplementary Fig. 4, Supplementary Table 1). Angptl1 and Angpt17's bindings to LAIR $1^{+}$human cord blood cells were relatively weak (Supplementary Fig. 5). We therefore focused on studying the binding of Angpt12 and Angptl5 to LILRB2 in subsequent experiments.

We determined whether LILRB2 was expressed on human HSCs. Flow cytometry and realtime RT-PCR analyses revealed that LILRB2 was expressed on the surface of 40-95\% of human cord blood $\mathrm{CD} 34^{+} \mathrm{CD} 38^{-} \mathrm{CD} 90^{+}$cells $(95 \%$ in the experiment shown in Fig. $2 \mathrm{~b}$; Supplementary Fig. 6); this population is enriched for HSCs. GST-Angpt15 treatment induced increased phosphorylation of calcium/calmodulin-dependent protein kinase CAMKII and CAMKIV in human cord blood mononuclear cells (Supplementary Fig. 7). It is of note that CAMKIV is required for maintenance of the potency of HSCs ${ }^{15}$. Suppression 
of LILRB2 expression with shRNAs effectively reduced Angptl binding (Supplementary Fig. 8). Importantly, the silencing of LILRB2 resulted in decreased repopulation of human cord blood HSCs as measured by reconstitution analysis in NOD/SCID mice (1\% repopulation from cultured knockdown cells compared to 17\% repopulation from cultured normal cells in medium STFA5; Fig. 2c). Together, these data indicate that the Angpt15 supports expansion of human cord blood HSCs ${ }^{1}$ in a process at least partially mediated by the surface receptor LILRB2.

The paired immunoglobulin-like receptor B (PirB) is the only mouse membrane ortholog of human LILRBs 16,17. Angpt12, Angpt13, and GST-Angpt15 bound to PirB as determined by flow cytometry (Fig. 3a, Supplementary Fig. 9) and by Co-IP (Fig. 3b, Supplementary Fig. 10). As were human cord blood HSCs, mouse HSCs were also enriched for PirB expression (Fig. 3c, Supplementary Fig. 11).

To study the function of PirB in mouse HSCs, we used PirB-deficient (PirBTM) mice ${ }^{18}$, in which four exons encoding the transmembrane domain and part of the intracellular domain were deleted. PirBTM cells freshly isolated from 3-week old of mice had significantly decreased CAMKIV phosphorylation, and binding of Angptl to PirB induced phosphorylation of PirB, recruitment of SHP-1 and SHP-2, and CAMKIV activation (Supplementary Figs. 12-13). These results suggest that certain Angptls may be the ligands of PirB that activate CAMKIV in vivo.

Because SHP-2 and CAMKIV are required for the repopulation of HSCs ${ }^{15,19}$, and the chemical inhibition of CAMKII, a homolog of CAMKIV, induces differentiation and suppresses proliferation of myeloid leukemia cells ${ }^{20}$, we sought to determine whether PirB was important for HSC activity. While the adult PirBTM mice have certain immune and neuronal defects, they are grossly normal in hematopoiesis ${ }^{16,18}$. Interestingly, competitive repopulation showed that PirBTM fetal liver HSCs had approximately 50\% decreased repopulation activity (Supplementary Fig. 14). Moreover, although Angpt12 and Angpt15 had little effect on ex vivo expansion of adult PirBTM HSCs, they supported ex vivo expansion of adult wild-type (WT) HSCs (Fig. 3d and Supplementary Fig. 14), as we previously demonstrated ${ }^{2}$. Collectively, our results indicate that Angptls bind human LILRB2 and mouse PirB to support HSC repopulation.

Based on our in silico analysis of a pool of 9004 samples described previously ${ }^{21}$, the level of LILRB2 mRNA is at least 4-fold higher in the human acute monoblastic and monocytic leukemia cells (M5 subtype of acute myeloid leukemia (AML)) than in other AML cells (Supplementary Fig. 15). Since human acute monoblastic and monocytic leukemia cells are often associated with rearrangement of MLL (a histone methyltransferase deemed a positive global regulator of gene transcription), we used a retroviral MLL-AF9 transplantation mouse model ${ }^{22,23}$ to further examine the role of PirB in regulation of AML development. WT or PirBTM donor Lin $^{-}$cells infected by retroviral MLL-AF9-IRES-YFP were used to induce AML as previously described ${ }^{22,23}$. We examined PirB expression in $\mathrm{YFP}^{+} \mathrm{Mac}-1^{+} \mathrm{Kit}^{+}$cells that may be enriched for AML initiating activity ${ }^{22,23}$, and found that about $80 \%$ $\mathrm{YFP}^{+} \mathrm{Mac}-1^{+} \mathrm{Kit}^{+}$cells were $\mathrm{PirB}^{+}$(Fig. 4a). We next investigated whether PirB was required for the induction of AML by MLL-AF9. Mice transplanted with MLL-AF9- 
transduced WT cells developed AML and died within approximately 5 weeks, whereas those transplanted with MLL-AF9-transduced PirBTM cells were resistant to the induction of MLL-AF9 and developed AML much more slowly (Fig. 4b, Supplementary Fig. 16). The significantly delayed development of the PirBTM leukemia was correlated with about 50\% lower numbers of white blood cells in circulation and a much less severe infiltration of myeloid leukemia cells into the liver and spleen (Fig. 4c-d). Consistently, PirB deficiency caused an approximately $50 \%$ reduction of $\mathrm{YFP}^{+} \mathrm{Mac}-1^{+} \mathrm{Kit}^{+}$cells in both bone marrow and peripheral blood (Fig. 4d). There were more $\mathrm{CD}^{+}$or $\mathrm{B} 220^{+}$cells in mice that received MLL-AF9-transduced PirBTM donor cells than in those given WT cells (Fig. 4d). These results demonstrate that PirB mediated signaling is associated with faster AML development and greater numbers of $\mathrm{YFP}^{+} \mathrm{Mac}-1^{+} \mathrm{Kit}^{+} \mathrm{AML}$ cells in vivo.

We further assessed whether PirB potentially regulates differentiation and self-renewal of AML cells. CFU assays showed that extrinsic Angptls stimulation led to increased CFU numbers in WT but not PirBTM AML cells, again indicating PirB directly mediates Angptls' effects (Supplementary Fig. 16d). In addition, WT AML cells formed mostly compact colonies, whereas PirBTM cells tended to form more diffuse ones (Fig. 4e). The formation of diffuse colonies indicates high differentiation potential ${ }^{24}$. The inhibition of differentiation of AML cells by PirB is accordant with previous reports that PirB inhibits differentiation of myeloid-derived suppressive cells ${ }^{25}$ and osteoclasts ${ }^{26}$, as well as our data showing that endogenous Angptls inhibit differentiation and increase replating efficiency of hematopoietic progenitors (Supplementary Fig. 17). Moreover, PirBTM primary CFUs were unable to form secondary colonies upon replating (Fig. 4f), suggesting that PirB supports self-renewal of AML CFU cells.

Finally, we analyzed the molecular signaling triggered by the binding of Angptls to PirB in AML cells. PirBTM AML cells had decreased phosphorylation of phosphatase SHP-2 (Fig. 4h), which is known to be associated with LILRB receptors and is an oncogene that supports leukemia development ${ }^{13,16,18,27}$. Angptls also stimulated SHP-2 phosphorylation (Supplementary Fig. 13c). Similar to untransformed PirBTM cells, PirBTM AML cells had decreased CAMKIV activation (data not shown). Furthermore, WT Mac- $1^{+} \mathrm{Kit}^{+}$cells had much greater expression of leukemia initiation/maintenance genes ${ }^{22,23}$ but dramatically decreased expression of myeloid differentiation genes as determined by DNA microarray analyses (Fig. 4g). Quantative RT-PCR confirmed the increased expression of several HoxA genes, Meis1, Eya1, Myb, and Mef2c in WT Mac- $1^{+} \mathrm{Kit}^{+}$cells than PirBTM counterparts (Supplementary Fig. 18); these genes are critical for initiation or maintenance of MLL rearranged AML 22,23. Similar to the MLL-AF9 model, the deficiency of PirB in the AML1ETO9a leukemia model led to decreased leukemia progenitors and increased differentiated cells (Supplementary Fig. 19). Collectively, these results suggest that the binding of Angptls to PirB promotes leukemia development, likely through inhibiting differentiation of AML cells.

LILRB2 or PirB is known to bind to other ligands including various MHC class I molecules ${ }^{28}$ and myelin inhibitors ${ }^{17}$. It will be important to investigate the in vivo context in which these different ligands bind LILRB and induce signaling. As Angptls can be abundantly expressed by many types of cells including those from endocrine organs ${ }^{11}$ and 
potential BM niche (endothelium and adipocytes ${ }^{9,11}$ ), and can be induced by hypoxia ${ }^{11}$, these secreted factors may have important direct and indirect effects on the activities of HSCs and leukemia stem cells in vivo. While the LILRB/PirB receptors were reported to suppress activation of differentiated immune cells and inhibit neurite outgrowth of neural cells ${ }^{16,17}$, they support HSC repopulation and inhibit differentiation of AML cells. This result suggests the significant importance of these "inhibitory receptors" in maintenance of stemness of normal stem cells and support of leukemia development. In contrast to the "stimulatory receptors" such as IFN receptors or toll-like receptors that activate and induce differentiation of HSCs upon inflammation ${ }^{29}$, LILRB2 or PirB may function as a sensor of inflammation through binding to the inflammatory Angptls ${ }^{12}$ and protect HSCs from excessive activation and exhaustion. Adult stem cells and cancer cells likely require both stimulatory receptors and inhibitory receptors to maintain the balance of their cell fates.

\section{Methods Summary}

Plasmid CMV-Kozak-human Ang1, Angptls 1, 2, 3, 4, 6, and 7 with FLAG tags at C-termini were used for transfection. Angptl2-FLAG was purified using M2 resin. Purified GSTAngpt15 was purchased from Abnova. Bacterially-expressed FLAG-Angpt12 and Angpt12FLAG were constructed in pET-26b(+) vector, and GST-Angptls-FLAG in pGEX vector, and expressed and purified from bacteria. MSCV-LILRB2-IRES-GFP or control retrovirus infected BAF3 cells, CMV-driven LILRAs, LILRBs, PirB, or LAIR1 transfected 293T cells, or human mononuclear cord blood cells were used in binding assays. See the Full Methods for detailed experimental methods for flow cytometry, co-IP, SPR, liquid-phase binding, culture, transplantation, CFU, and GSEA analyses. Mice were maintained at the UT Southwestern Medical Center animal facility. All animal experiments were performed with the approval of UT Southwestern Committee on Animal Care.

\section{Methods}

Mice

C57 BL/6 CD45.2 and CD45.1 mice, or NOD/SCID mice were purchased from the UT Southwestern Medical Center animal breeding core facility. The PirBTM mice ${ }^{18}$ were obtained from MMRRC. The PirB knockout mice ${ }^{31}$ were a gift from Dr. T. Takai at Tohoku University. Mice were maintained at the UT Southwestern Medical Center animal facility. All animal experiments were performed with the approval of UT Southwestern Committee on Animal Care.

\section{Plasmids and proteins}

Plasmid CMV-Kozak-human Ang1, Angptls 1, 2, 3, 4, 6, and 7 with FLAG tags at C-termini were transfected into $293 \mathrm{~T}$ cells using Lipofectamine 2000, and the conditioned medium at $48 \mathrm{~h}$ was collected and different Angptl proteins were adjusted to the same level for flow cytometry based binding experiments. Angptl2-FLAG was purified using M2 resin. Purified GST-Angpt15 was purchased from Abnova. Bacterially-expressed Flag-Angpt12 and Angpt12-Flag were constructed in pET-26b(+) vector, and GST-Angptls-FLAG in pGEX vector, and expressed and purified from bacteria. MSCV-LILRB2-IRES-GFP or control 
retrovirus infected BAF3 cells, or CMV-driven LILRAs, LILRBs, PirB, or LAIR1 transfected 293T cells harvested at $48 \mathrm{~h}$, or mononuclear human cord blood cells were incubated with $\mathrm{Fc}$ block and equal amounts of different FLAG-tagged Angptls at $4^{\circ} \mathrm{C}$ for 60 min, followed by staining with anti-Flag-APC and propidium iodide. Anti-LILRB2-PE was used as indicated. Cells were analyzed using either a FACSCalibur or FACSAria instrument (Becton Dickinson).

\section{Antibodies and shRNAs}

Flow cytometry antibodies anti-CD34-FITC, anti-CD38-PE, anti-CD90-PE/Cy5.5, biotinylated lineage cocktail, anti-Kit-APC, anti-Sca-1-FITC, anti-Mac-1-APC, anti-Gr-1$\mathrm{PE}$, anti-CD3-APC, and anti-B220-PE were purchased from BD Biosciences and used as described $4,9,32,33$. The manufacturers and catalog numbers for other antibodies are as follows: anti-LILRB1, Biolegend (33707); anti-LILRB2, eBioscience (12-5149); antiLILRB3, eBioscience (12-5159); anti-LILRB4, eBiosciene (12-5139); anti-LILRB5, R\&D Systems (AF3065); anti-PirB-PE, R\&D Systems (FAB2754P); anti-human LAIR1-PE, BD Pharmingen (550811); anti-mouse LAIR1-PE, eBioscience (12-3051); anti-FLAG-APC, Prozyme (PJ255); anti-pCAMKII, Abcam (ab32678); anti-pCAMKIV, Santa Cruz (sc-28443-R); anti-CAMKII, Cell Signaling (4436); anti-CAMKIV, Cell Signaling (4032); anti-Angptl5, Abcam (ab57240); anti-PirB, BD Pharmingen (550348) for co-IP of PirB; anti-SHP-2, Cell Signaling (3397S) for co-IP of SHP-2; and anti-hFc, Jackson ImmunoResearch (109-036-098). Combinations of multiple lentivirus-expressed shRNAs for inhibition of LILRB2 (hairpin sequences:

TGCTGTTGACAGTGAGCGCCAGCTTGACCCTCAGACGGAATAGTGAAGCCACAG ATGTATTCCGTCTGAGGGTCAAGCTGTTGCCTACTGCCTCGGA and TGCTGTTGACAGTGAGCGCACGACCAGAGCTTGTGAAGAATAGTGAAGCCACA GATGTATTCTTCACAAGCTCTGGTCGTATGCCTACTGCCTCGGA), Angptl1 (TGCTGTTGACAGTGAGCGCCTCGTGTTACTCAACTCTATATAGTGAAGCCACAG ATGTATATAGAGTTGAGTAACACGAGATGCCTACTGCCTCGGA, TGCTGTTGACAGTGAGCGAAGAGACACTCGCCAATTTAAATAGTGAAGCCACAG ATGTATTTAAATTGGCGAGTGTCTCTCTGCCTACTGCCTCGGA, TGCTGTTGACAGTGAGCGACCAATTTAAATGACACAGAACTAGTGAAGCCACAG ATGTAGTTCTGTGTCATTTAAATTGGCTGCCTACTGCCTCGGA), Angpt12 (TGCTGTTGACAGTGAGCGCCACAGAGTTCTTGGAATAAAATAGTGAAGCCACA GATGTATTTTATTCCAAGAACTCTGTGATGCCTACTGCCTCGGA, TGCTGTTGACAGTGAGCGACACAGCAGCGGCAGAAGCTTATAGTGAAGCCACA GATGTATAAGCTTCTGCCGCTGCTGTGGTGCCTACTGCCTCGGA, TGCTGTTGACAGTGAGCGCCAGATGGAGGCTGGACAGTAATAGTGAAGCCACA GATGTATTACTGTCCAGCCTCCATCTGATGCCTACTGCCTCGGA), Angptl3 (TGCTGTTGACAGTGAGCGACTCAGAAGGACTAGTATTCAATAGTGAAGCCACA GATGTATTGAATACTAGTCCTTCTGAGCTGCCTACTGCCTCGGA, TGCTGTTGACAGTGAGCGCCAGCATAGTCAAATAAAAGAATAGTGAAGCCACA GATGTATTCTTTTATTTGACTATGCTGTTGCCTACTGCCTCGGA, TGCTGTTGACAGTGAGCGATACATATAAACTACAAGTCAATAGTGAAGCCACAG ATGTATTGACTTGTAGTTTATATGTAGTGCCTACTGCCTCGGA), Angpt14 (TGCTGTTGACAGTGAGCGCCACAGAGTTCTTGGAATAAAATAGTGAAGCCACA 
GATGTATTTTATTCCAAGAACTCTGTGATGCCTACTGCCTCGGA, TGCTGTTGACAGTGAGCGACACAGCAGCGGCAGAAGCTTATAGTGAAGCCACA GATGTATAAGCTTCTGCCGCTGCTGTGGTGCCTACTGCCTCGGA, TGCTGTTGACAGTGAGCGCCAGATGGAGGCTGGACAGTAATAGTGAAGCCACA GATGTATTACTGTCCAGCCTCCATCTGATGCCTACTGCCTCGGA), Angpt15 (TGCTGTTGACAGTGAGCGATAGAAGATGGATCTAATGCAATAGTGAAGCCACA GATGTATTGCATTAGATCCATCTTCTACTGCCTACTGCCTCGGA, TGCTGTTGACAGTGAGCGAATGGTTTAGATTGCACTGATATAGTGAAGCCACAG ATGTATATCAGTGCAATCTAAACCATGTGCCTACTGCCTCGGA, TGCTGTTGACAGTGAGCGATACGGACTCTTCAGTAGTTAATAGTGAAGCCACAG ATGTATTAACTACTGAAGAGTCCGTAGTGCCTACTGCCTCGGA), Angptl6 (TGCTGTTGACAGTGAGCGCCACTACCTGGCAGCACTATAATAGTGAAGCCACA GATGTATTATAGTGCTGCCAGGTAGTGATGCCTACTGCCTCGGA, TGCTGTTGACAGTGAGCGAGAGGCAAGATGGTTCAGTCAATAGTGAAGCCACA GATGTATTGACTGAACCATCTTGCCTCCTGCCTACTGCCTCGGA, TGCTGTTGACAGTGAGCGACCCAGAGAGACCAGACCCAGATAGTGAAGCCACA GATGTATCTGGGTCTGGTCTCTCTGGGGTGCCTACTGCCTCGGA), and Angptl7 (TGCTGTTGACAGTGAGCGCCCGGGACTGGAAGCAGTACAATAGTGAAGCCACA GATGTATTGTACTGCTTCCAGTCCCGGTTGCCTACTGCCTCGGA, TGCTGTTGACAGTGAGCGCCCGCATCTCTGGAGTGTATAATAGTGAAGCCACAG ATGTATTATACACTCCAGAGATGCGGTTGCCTACTGCCTCGGA, TGCTGTTGACAGTGAGCGCGGACTGAGAAACAGCCTATAATAGTGAAGCCACA GATGTATTATAGGCTGTTTCTCAGTCCTTGCCTACTGCCTCGGA) were purchased from Open Biosystems and used for knockdown experiments. The specificity of LILRB2 $\mathrm{mAb}$ is confirmed by comparison of binding to all tested LILRA/Bs on transfected 293T cells. The specificities of other anti-LILRBs, anti-PirB, and anti-LAIR1 were confirmed by staining the respective cDNA overexpressed 293T cells.

\section{Co-immunoprecipitation}

For in vivo co-IP, 293 cells were transiently co-transfected with plasmids encoding LILRB2ECD-hFc, PirB-ECD-hFc, or Tie-2-ECD-hFc and FLAG-tagged Angptl2 or untagged Angpt15. Protein A beads were added to conditioned medium collected at $48 \mathrm{~h}$ after transfection, and proteins were detected by anti-FLAG or anti-Angpt15 by western blot. For in vitro co-IP, purified Angpt12-FLAG or GST-Angpt15 was incubated with purified LILRB2-ECD-hFc or Tie2-ECD-hFc in PBS with $0.1 \%$ BSA and $0.1 \%$ NP-40 for $2 \mathrm{~h}$ followed by immunoprecipitated with protein A beads and western blotting.

\section{Liquid-phase binding Assay}

Specific binding of radiolabeled GST-Angpt15 to BAF3 stably infected with MSCVLILRB2-IRES-GFP (as LILRB2-BAF3 cells) was performed similarly as we performed before ${ }^{30}$. Briefly, $6 \times 10^{6}$ LILRB2-BAF3 cells were incubated with ${ }^{125}$ I-GST-Angpt15 (0.1 $-100 \mathrm{nM}$ ) in $200 \mu \mathrm{PBS} / 1 \% \mathrm{BSA}$ for $3 \mathrm{~h}$ at $25^{\circ} \mathrm{C}$. Nonspecific binding on normal BAF3 cells was subtracted. In competition assay, $2.5 \times 10^{6}$ LILRB2-BAF3 or BAF3 cells were incubated with unlabeled GST-Angpt15 $(0.1-100 \mathrm{nM})$ in $200 \mu \mathrm{l}$ PBS/1\% BSA for $1 \mathrm{~h}$ at 25 ${ }^{\circ} \mathrm{C}$, followed by addition of $5 \mathrm{nM}$ of ${ }^{125} \mathrm{I}$-GST-Angpt15 for $4 \mathrm{~h}$ incubation. After incubation, 
the cells were washed twice by centrifugation, resuspended in ice-cold PBS with $1 \%$ BSA and then measured in scintillation counter.

\section{Cell culture and infection}

BaF3 cells were grown in RPMI medium 1640 with $10 \%$ FBS and 10\% Wehi conditioned cell medium. Human embryonic kidney 293T cells were grown in DMEM with 10\% FBS.

For mouse $\mathrm{HSC}$ culture, indicated numbers of $\mathrm{BM} \mathrm{Lin}{ }^{-} \mathrm{Sca}-1^{+} \mathrm{Kit}^{+} \mathrm{CD} 34^{-} \mathrm{Flk}-2^{-}$cells or fetal liver $\mathrm{Lin}{ }^{-} \mathrm{Sca}-{ }^{+} \mathrm{Kit}^{+}$cells isolated from 8-10 week old C57BL/6 CD45.2 mice were plated in one well of a U-bottom 96-well plate (Corning) with $200 \mu \mathrm{l}$ of the indicated medium essentially as we described previously ${ }^{4,9}$. Cells were cultured at $37^{\circ} \mathrm{C}$ in $5 \% \mathrm{CO}_{2}$ and indicated levels of $\mathrm{O}_{2}$. For the purpose of competitive transplantation, we pooled cells from 12 culture wells and mixed them with competitor/supportive cells before the indicated numbers of cells were transplanted into each mouse. For western blotting, 3-week old mouse spleen cells were cultured overnight in DME supplemented with $0.1 \%$ BSA, followed by treatment with indicated amount of Angptls. Human mononuclear cord blood cells were cultured in DME containing 10\% FBS overnight followed by starvation in serum-free DME for $4 \mathrm{~h}$ before Angptl stimulation.

The infection of $\mathrm{Lin}^{-}$cells by MSCV-MLL-AF9-IRES-YFP and MSCV-AML1-ETO9aIRES-GFP was performed following procedures described by the Armstrong and Cleary laboratories ${ }^{22,23}$ and Zhang laboratory ${ }^{34}$, respectively. Briefly, we incubated Lin $^{-}$cells overnight in medium with $10 \%$ FBS, $20 \mathrm{ng} / \mathrm{mL}$ SCF, $20 \mathrm{ng} / \mathrm{ml} \mathrm{IL-3,} \mathrm{and} 10 \mathrm{ng} / \mathrm{mL}$ IL-6, followed by spin infection with retroviral supernatant in the presence of $4 \mu \mathrm{g} / \mathrm{mL}$ polybrene. Infected cells $(300,000)$ were transplanted into lethally irradiated (1000 rad) C57BL/6 mice by retro-orbital injection.

For human cell culture, fresh and cryopreserved human cord blood cells were obtained from UT Southwestern Parkland Hospital through approved IRB protocol 042008-033. CD34+ cells were isolated by AutoMACS and cultured essentially as we described ${ }^{1,32}$. CD133 ${ }^{+}$ cells were purchased from AllCell Inc. Lentiviral infection by shRNAs for LILRB2 or Angptls was performed as recommended by Open Biosystems.

\section{Flow cytometry and reconstitution analysis}

Donor mouse bone marrow cells were isolated from 8-10 week old C57BL/6 CD45.2 mice. $\mathrm{BM} \mathrm{Lin}{ }^{-} \mathrm{Sca}-1^{+} \mathrm{Kit}^{+} \mathrm{CD} 34^{-} \mathrm{Flk}-2^{-}$cells were isolated by staining with a biotinylated lineage cocktail (anti-CD3, anti-CD5, anti-B220, anti-Mac-1, anti-Gr-1, anti-Ter119, and anti-7-4; Stem Cell Technologies) followed by streptavidin-PE/Cy5.5, anti-Sca-1-FITC, anti-KitAPC, anti-CD34-PE, and anti-Flk-2-PE. The indicated numbers of mouse CD45.2 donor cells were mixed with $1 \times 10^{5}$ freshly isolated CD45.1 competitor bone marrow cells, and the mixture injected intravenously via the retro-orbital route into each of a group of 6-9 week old CD45.1 mice previously irradiated with a total dose of $10 \mathrm{~Gy}$. To measure reconstitution of transplanted mice, peripheral blood was collected at the indicated times post-transplant and CD45.1 $1^{+}$and CD45.2 $2^{+}$cells in lymphoid and myeloid compartments were measured as we described ${ }^{4,9,32}$. The analyses of Mac-1, Kit, Gr-1, CD3, B220 
populations in AML blood or bone marrow were performed by using anti-Mac-1-APC, antiKit-PE, anti-Gr-1-PE, anti-CD3-APC, and anti-B220-PE.

Uncultured or cultured progenies of human cells were pooled together and the indicated portions were injected intravenously via the retro-orbital route into sub-lethally irradiated (250 rad) 6-8 week old NOD/SCID mice. Eight weeks after transplantation, bone marrow nucleated cells from transplanted animals were analyzed by flow cytometry for the presence of human cells as we described 1,32 .

\section{CFU assays}

Two thousand $\mathrm{YFP}^{+} \mathrm{Mac}-1^{+} \mathrm{Kit}^{+} \mathrm{BM}$ cells from AML mice were plated in methylcellulose (M3534, Stem Cell Technologies) for CFU-GM assays, according to the manufacturer's protocols and our previously published protocol ${ }^{35}$. After 7 days, 2000 cells from initially plated three dishes were used for secondary replating.

\section{Surface plasmon resonance}

Biacore 2000 and CM5 chips were used to analyze binding of purified Angptls to the LILRB2 extracellular domain fused to $\mathrm{hFc}$, using a method similar to that previously described ${ }^{36}$. Recombinant protein A (Pierce) was pre-immobilized in two flow cells $(\sim 2,000 \mathrm{RU})$ using the amine-coupling kit from GE. LILRB2-hFc was injected into one of the flow cells to be captured by the protein A to reach $\sim 300$ response units (RU). GSTAngpt15 was injected over the immobilized LILRB2 in HBS-EP (GE) containing $0.01 \mathrm{M}$ HEPES (pH 7.4), $0.15 \mathrm{M} \mathrm{NaCl}, 0.005 \%$ polysorbate 20 . Each binding sensorgram from the sample flow cell, containing a captured LILRB2-hFc, was corrected for the protein A coupled cell control. Following each injection of an antigen solution, which induced the binding reaction, and the dissociation period during which the running buffer was infused, the protein A surface was regenerated by the injection of the regeneration solution containing $10 \mathrm{mM} \mathrm{Na}_{3} \mathrm{PO}_{4}(\mathrm{pH} 2.5$ ) and $500 \mathrm{mM} \mathrm{NaCl}$. All captured LILRB2-hFc, with and without Angptl5 bound, was completely removed, and another cycle begun. All measurements were performed at $25^{\circ} \mathrm{C}$ with a flow rate of $30 \mu \mathrm{L} / \mathrm{min}$.

\section{GSEA analysis}

Gene set enrichment analysis ${ }^{37}$ was performed using GSEA v2.0 software (http:// www.broadinstitute.org/gsea/index.jsp) with 1,000 phenotype permutations, and normalized enrichment score (NES) and false discovery rate q-value (FDR q-val) were calculated. Leukemia-stem-cell and macrophage development gene sets were obtained from the indicated publication ${ }^{23,38}$.

\section{Statistics}

Two-tailed student t-test was performed to evaluate significance between experimental groups, unless otherwise is indicated. The survival rates of the two groups will be analyzed using a log-rank test. 


\section{Supplementary Material}

Refer to Web version on PubMed Central for supplementary material.

\section{Acknowledgments}

We thank Dr. Scott Armstrong for the MSCV-MLL-AF9-IRES-YFP construct, Dr. Helen Hobbs for the CMVhAngpt16-FLAG plasmid, Dr. Toshiyuki Takai for providing the PirB knockout mice to Dr. Shu-Hsia Chen, Dr. Xian-Jin Xie for binding analysis, and UTSW Genomics and Microarray Core facility for DNA array experiments. Support to C. C. Z. was from NIH grant K01 CA 120099, American Society of Hematology Junior Faculty Award, DOD PR093256, CPRIT RP100402, and the Gabrielle's Angel Foundation.

\section{References}

1. Zhang CC, Kaba M, Iizuka S, Huynh H, Lodish HF. Angiopoietin-like 5 and IGFBP2 stimulate ex vivo expansion of human cord blood hematopoietic stem cells as assayed by NOD/SCID transplantation. Blood. 2008; 111:3415-3423. doi:blood-2007-11-122119 [pii] 10.1182/ blood-2007-11-122119 [doi]. [PubMed: 18202223]

2. Zhang CC, et al. Angiopoietin-like proteins stimulate ex vivo expansion of hematopoietic stem cells. Nat Med. 2006; 12:240-245. [PubMed: 16429146]

3. Zhang CC, Lodish HF. Cytokines regulating hematopoietic stem cell function. Curr Opin Hematol. 2008; 15:307-311. doi:10.1097/MOH.0b013e3283007db5 [doi]00062752-200807000-00006 [pii]. [PubMed: 18536567]

4. Huynh H, et al. IGFBP2 secreted by a tumorigenic cell line supports ex vivo expansion of mouse hematopoietic stem cells. Stem Cells. 2008; 26:1628-1635. [PubMed: 18369099]

5. Chou S, Lodish HF. Fetal liver hepatic progenitors are supportive stromal cells for hematopoietic stem cells. Proc Natl Acad Sci U S A. 2010; 107:7799-7804. doi:1003586107 [pii] 10.1073/pnas. 1003586107 [doi]. [PubMed: 20385801]

6. Lin M, Zon LI. Genetic analyses in zebrafish reveal that angiopoietin-like proteins 1 and 2 are required for HSC development during embryogenesis. ASH 50th Annula Meeting Abstract. 2008; 729:186.

7. Khoury M, et al. Mesenchymal Stem Cells Secreting Angiopoietin-Like-5 Support Efficient Expansion of Human Hematopoietic Stem Cells Without Compromising Their Repopulating Potential. Stem Cells Dev. 2011 doi:10.1089/scd.2010.0456.

8. Drake AC, et al. Human CD34 CD133 Hematopoietic Stem Cells Cultured with Growth Factors Including Angpt15 Efficiently Engraft Adult NOD-SCID Il2rgamma (NSG) Mice. PLoS One. 2011; 6:e18382. doi:10.1371/journal.pone.0018382. [PubMed: 21559522]

9. Zheng J, Huynh H, Umikawa M, Silvany R, Zhang CC. Angiopoietin-like protein 3 supports the activity of hematopoietic stem cells in the bone marrow niche. Blood. 2011; 117:470-479. doi: 10.1182/blood-2010-06-291716. [PubMed: 20959605]

10. Zheng J, et al. Ex vivo expanded hematopoietic stem cells overcome the MHC barrier in allogeneic transplantation. Cell Stem Cell. 2011; 9:119-130. doi:10.1016/j.stem.2011.06.003. [PubMed: 21816363]

11. Hato T, Tabata M, Oike Y. The role of angiopoietin-like proteins in angiogenesis and metabolism. Trends Cardiovasc Med. 2008; 18:6-14. doi:S1050-1738(07)00241-1 [pii]10.1016/j.tcm. 2007.10.003 [doi]. [PubMed: 18206803]

12. Tabata M, et al. Angiopoietin-like protein 2 promotes chronic adipose tissue inflammation and obesity-related systemic insulin resistance. Cell Metab. 2009; 10:178-188. doi:10.1016/j.cmet. 2009.08.003. [PubMed: 19723494]

13. Barrow AD, Trowsdale J. The extended human leukocyte receptor complex: diverse ways of modulating immune responses. Immunol Rev. 2008; 224:98-123. doi:10.1111/j.1600-065X. 2008.00653.x. [PubMed: 18759923]

14. Meyaard L. LAIR and collagens in immune regulation. Immunol Lett. 2010; 128:26-28. doi: 10.1016/j.imlet.2009.09.014. [PubMed: 19836418] 
15. Kitsos CM, et al. Calmodulin-dependent protein kinase IV regulates hematopoietic stem cell maintenance. J Biol Chem. 2005; 280:33101-33108. doi:M505208200 [pii]10.1074/ jbc.M505208200 [doi]. [PubMed: 16020540]

16. Takai T, Nakamura A, Endo S. Role of PIR-B in autoimmune glomerulonephritis. J Biomed Biotechnol. 2011; 2011:275302. doi:10.1155/2011/275302. [PubMed: 20976309]

17. Atwal JK, et al. PirB is a functional receptor for myelin inhibitors of axonal regeneration. Science. 2008; 322:967-970. doi:10.1126/science.1161151. [PubMed: 18988857]

18. Syken J, Grandpre T, Kanold PO, Shatz CJ. PirB restricts ocular-dominance plasticity in visual cortex. Science. 2006; 313:1795-1800. doi:10.1126/science.1128232. [PubMed: 16917027]

19. Chan RJ, et al. Shp-2 heterozygous hematopoietic stem cells have deficient repopulating ability due to diminished self-renewal. Exp Hematol. 2006; 34:1230-1239. doi:10.1016/j.exphem. 2006.04.017. [PubMed: 16939816]

20. Si J, Collins SJ. Activated Ca2+/calmodulin-dependent protein kinase IIgamma is a critical regulator of myeloid leukemia cell proliferation. Cancer Res. 2008; 68:3733-3742. doi: 10.1158/0008-5472.can-07-2509. [PubMed: 18483256]

21. Lukk M, et al. A global map of human gene expression. Nat Biotechnol. 2010; 28:322-324. doi: 10.1038/nbt0410-322. [PubMed: 20379172]

22. Krivtsov AV, et al. Transformation from committed progenitor to leukaemia stem cell initiated by MLL-AF9. Nature. 2006; 442:818-822. doi:nature04980 [pii]10.1038/nature04980 [doi]. [PubMed: 16862118]

23. Somervaille TC, Cleary ML. Identification and characterization of leukemia stem cells in murine MLL-AF9 acute myeloid leukemia. Cancer Cell. 2006; 10:257-268. doi:S1535-6108(06)00276-5 [pii]10.1016/j.ccr.2006.08.020 [doi]. [PubMed: 17045204]

24. Lavau C, Szilvassy SJ, Slany R, Cleary ML. Immortalization and leukemic transformation of a myelomonocytic precursor by retrovirally transduced HRX-ENL. EMBO J. 1997; 16:4226-4237. [PubMed: 9250666]

25. Ma G, et al. Paired immunoglobin-like receptor-B regulates the suppressive function and fate of myeloid-derived suppressor cells. Immunity. 2011; 34:385-395. doi:10.1016/j.immuni. 2011.02.004. [PubMed: 21376641]

26. Mori Y, et al. Inhibitory immunoglobulin-like receptors LILRB and PIR-B negatively regulate osteoclast development. J Immunol. 2008; 181:4742-4751. [PubMed: 18802077]

27. Chan RJ, Feng GS. PTPN11 is the first identified proto-oncogene that encodes a tyrosine phosphatase. Blood. 2007; 109:862-867. doi:10.1182/blood-2006-07-028829. [PubMed: 17053061]

28. Shiroishi M, et al. Human inhibitory receptors Ig-like transcript 2 (ILT2) and ILT4 compete with CD8 for MHC class I binding and bind preferentially to HLA-G. Proc Natl Acad Sci U S A. 2003; 100:8856-8861. doi:10.1073/pnas.1431057100. [PubMed: 12853576]

29. Baldridge MT, King KY, Goodell MA. Inflammatory signals regulate hematopoietic stem cells. Trends Immunol. 2011; 32:57-65. doi:10.1016/j.it.2010.12.003. [PubMed: 21233016]

30. Zhang CC, Krieg S, Shapiro DJ. HMG-1 stimulates estrogen response element binding by estrogen receptor from stably transfected HeLa cells. Mol Endocrinol. 1999; 13:632-643. [PubMed: 10194768]

\section{References}

31. Ujike A, et al. Impaired dendritic cell maturation and increased $\mathrm{T}(\mathrm{H}) 2$ responses in PIR-B(-/-) mice. Nat Immunol. 2002; 3:542-548. doi:10.1038/ni801. [PubMed: 12021780]

32. Zheng J, et al. Ex vivo expanded hematopoietic stem cells overcome the MHC barrier in allogeneic transplantation. Cell Stem Cell. 2011 In press.

33. Simsek T, et al. The Distinct Metabolic Profile of Hematopoietic Stem Cells Reflects Their Location in a Hypoxic Niche. Cell Stem Cell. 2010; 7:380-390. doi:S1934-5909(10)00347-4 [pii]10.1016/j.stem.2010.07.011 [doi]. [PubMed: 20804973]

34. Yan $M$, et al. A previously unidentified alternatively spliced isoform of $t(8 ; 21)$ transcript promotes leukemogenesis. Nat Med. 2006; 12:945-949. doi:10.1038/nm1443. [PubMed: 16892037] 
35. Zhang CC, Steele AD, Lindquist S, Lodish HF. Prion protein is expressed on long-term repopulating hematopoietic stem cells and is important for their self-renewal. Proc Natl Acad Sci U S A. 2006; 103:2184-2189. [PubMed: 16467153]

36. Luo Y, Lu Z, Raso SW, Entrican C, Tangarone B. Dimers and multimers of monoclonal IgG1 exhibit higher in vitro binding affinities to Fcgamma receptors. MAbs. 2009; 1:491-504. [PubMed: 20065648]

37. Subramanian A, et al. Gene set enrichment analysis: a knowledge-based approach for interpreting genome-wide expression profiles. Proc Natl Acad Sci U S A. 2005; 102:15545-15550. doi: 10.1073/pnas.0506580102. [PubMed: 16199517]

38. Zuber J, et al. RNAi screen identifies Brd4 as a therapeutic target in acute myeloid leukaemia. Nature. 2011; 478:524-528. doi:10.1038/nature10334. [PubMed: 21814200] 

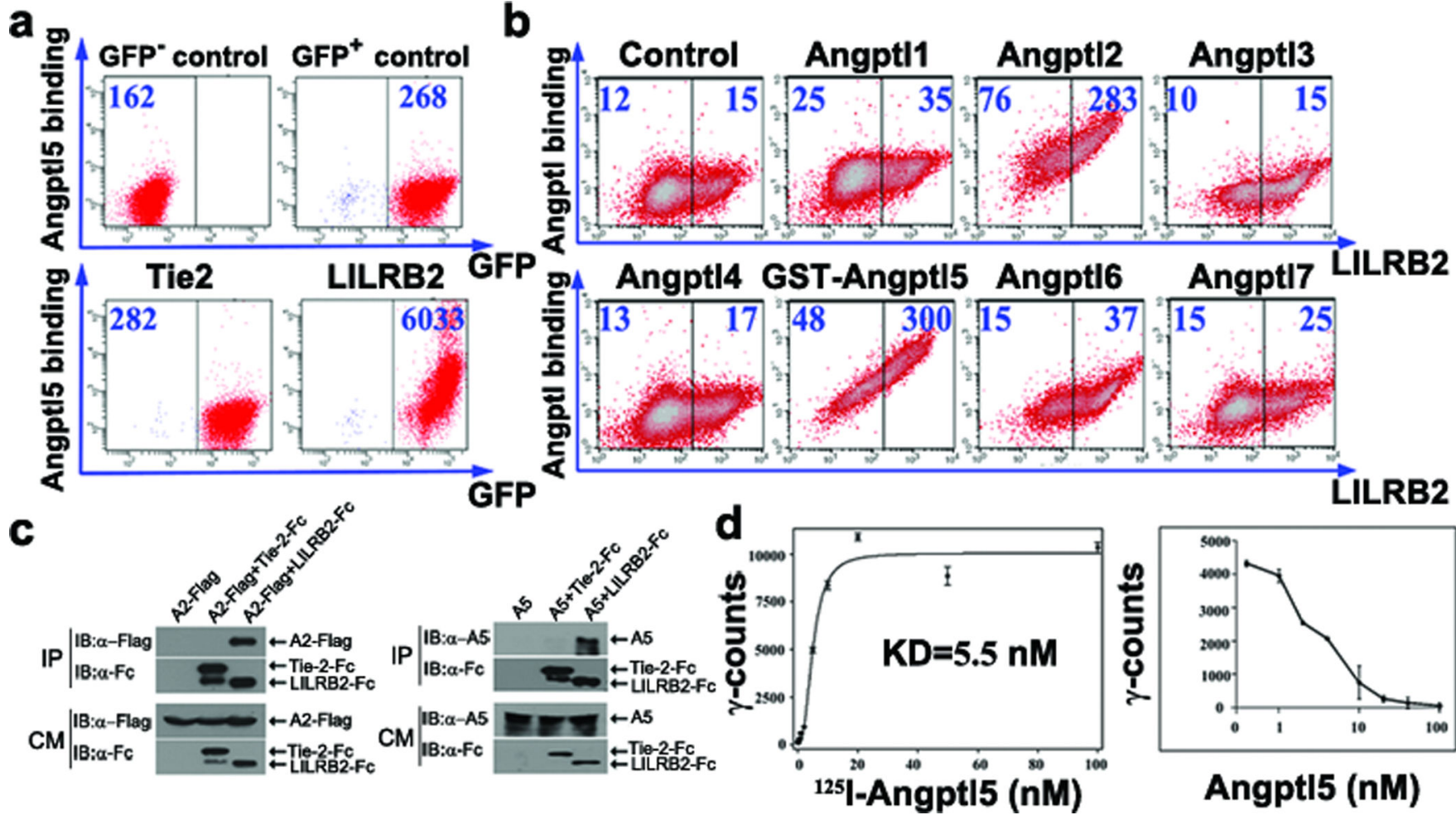

Figure 1. Cell surface LILRB2 binds to Angptls

a, Flow cytometry analysis of GST-Angptl5-FLAG binding to uninfected BAF3 cells or MSCV-GFP, MSCV-Tie2-GFP, or MSCV-LILRB2-GFP stably infected BAF3 cells. MFIs were indicated. b, Flow cytometry analysis of indicated FLAG-tagged Angptls binding to LILRB2 transfected 293T cells. c, Angptl2 and Angptl5 bound to the extracellular domain of LILRB2 but not Tie-2 in conditioned medium (CM) of co-transfected 293T cells (IB, immunoblotting; IP, immunoprecipitation). d-e, Concentration dependent specific (d) and competitive (e) ${ }^{125}$ I-GST-Angpt15 binding to LILRB2 stably expressed BAF3 cells $(\mathrm{n}=3)$. Error bars, s.e.m. 


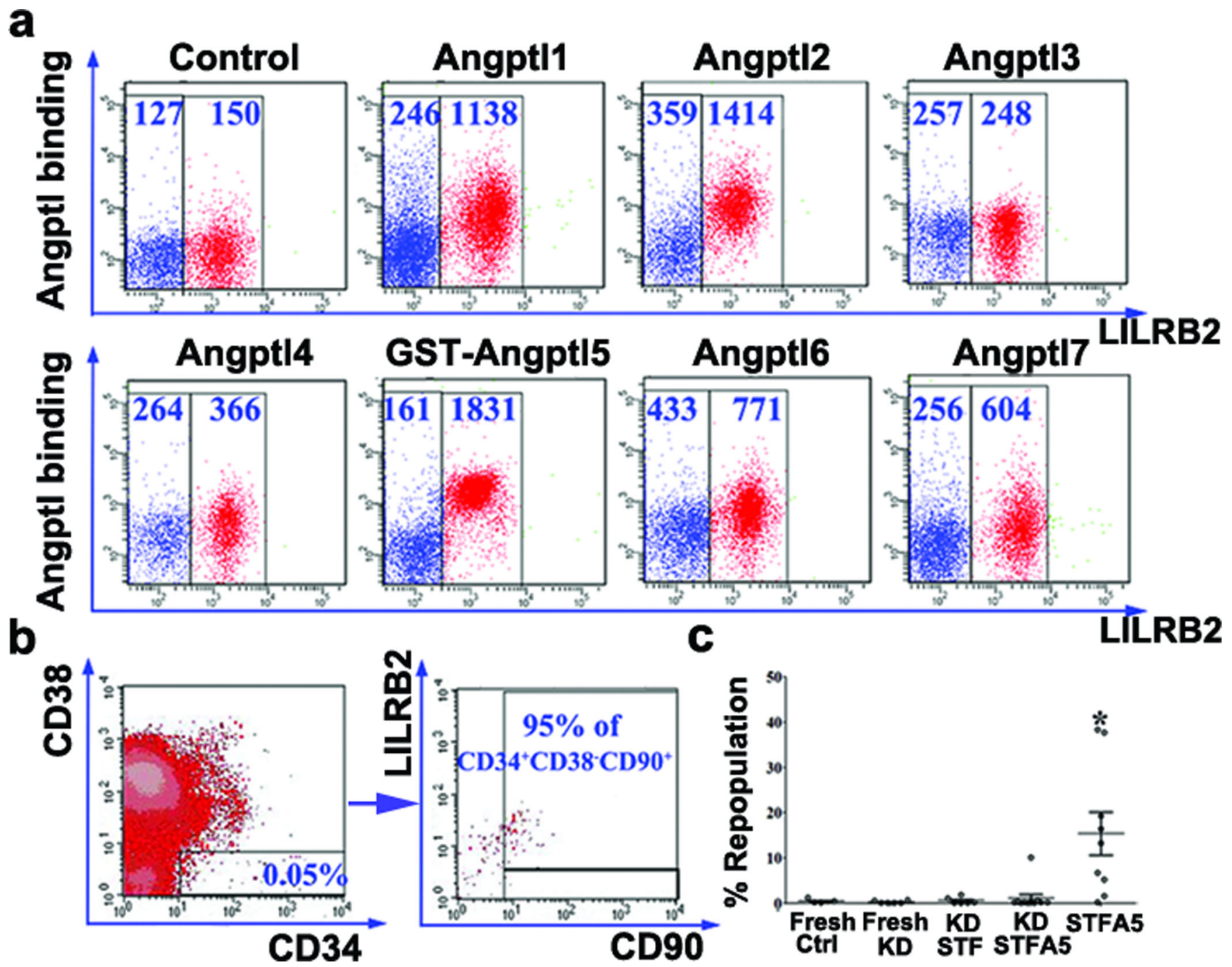

Figure 2. LILRB2 mediates the effect of Angptl in supporting the repopulation of human cord blood HSCs

a, Flow cytometry analysis of indicated FLAG-tagged Angptls binding to LILRB2 ${ }^{+}$human cord blood mononuclear cells (FACSAria). MFIs were indicated. b, Representative flow cytometry plots for the co-staining of CD34, CD38, CD90, and LILRB2 in human cord blood mononuclear cells (FACSCalibur). c, Human cord blood CD34 ${ }^{+}$cells infected with LILRB2 shRNA-encoding virus (KD) or control scramble shRNA virus were transplanted into sublethally irradiated NOD/SCID mice before or after culture for $10 \mathrm{~d}$. SCF+TPO + Flt3L (STF) or STF+Angptl5 (STFA5) was used in the culture. Shown is the human donor repopulation after 2 months $(\mathrm{n}=5-11){ }^{*} \mathrm{p}<0.05$. Error bars, s.e.m. 

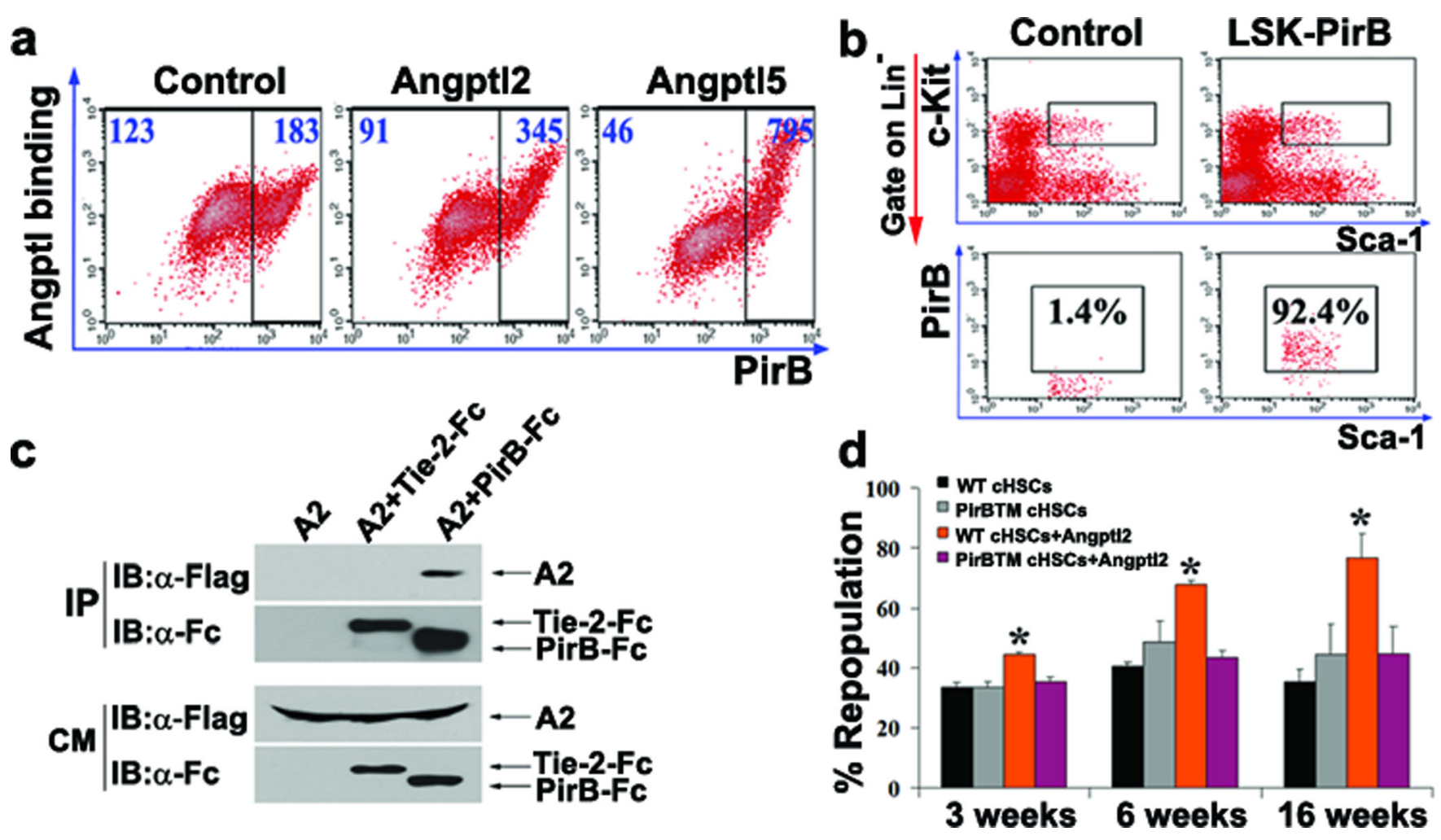

Figure 3. Angptls bind PirB and support the repopulation of mouse HSCs

a, Flow cytometry analysis of FLAG-Angpt12 or GST-Angpt15-FLAG binding to PirB transfected 293T cells. b, Angptl2 binds to the extracellular domain of PirB but not Tie-2 in the conditioned medium of co-transfected 293 T cells. c, PirB is expressed on mouse BM $\mathrm{Lin}^{-} \mathrm{Sca}-1^{+} \mathrm{Kit}^{+}$cells. Isotype control on left. $\mathbf{d}$, Competitive reconstitution of 8-day cultured progenies of input equivalent $250 \mathrm{Lin}^{-} \mathrm{Sca}-1^{+} \mathrm{Kit}^{+} \mathrm{CD} 34^{-} \mathrm{Flk} 2^{-} \mathrm{BM}$ HSCs from WT or PirBTM donors $(n=5)$. SCF, TPO, and FGF-1, with or without Angptl2, were used in culture. $* \mathrm{p}<0.05$. Error bars, s.e.m. 

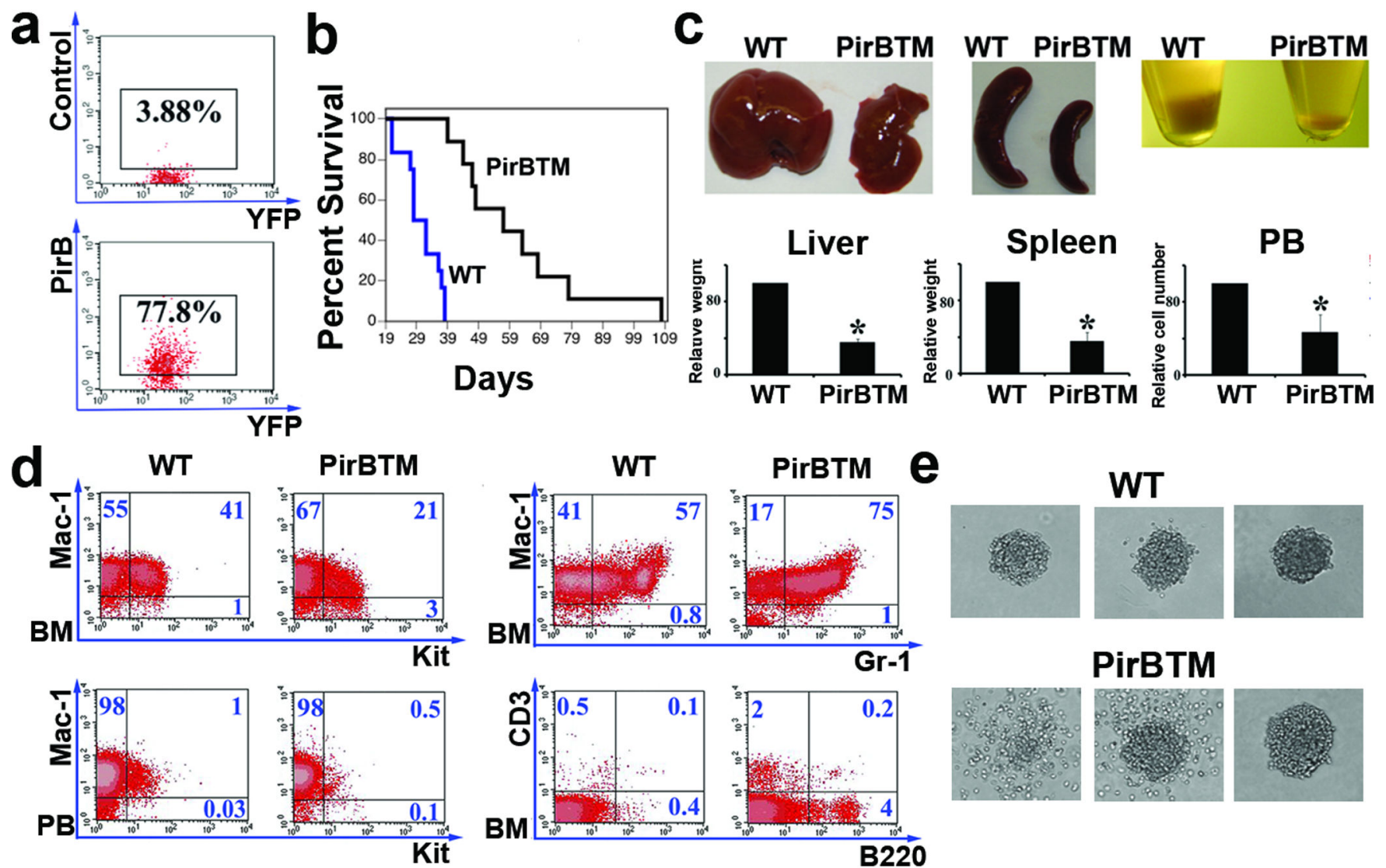

PirBTM

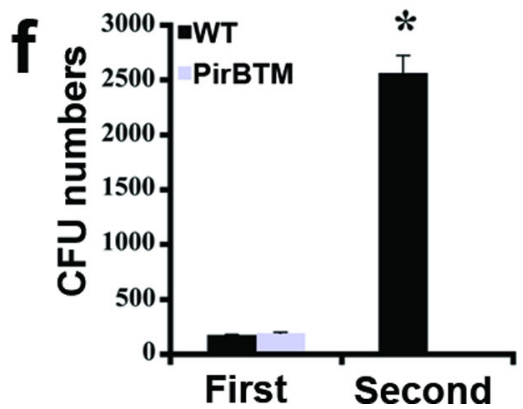

g
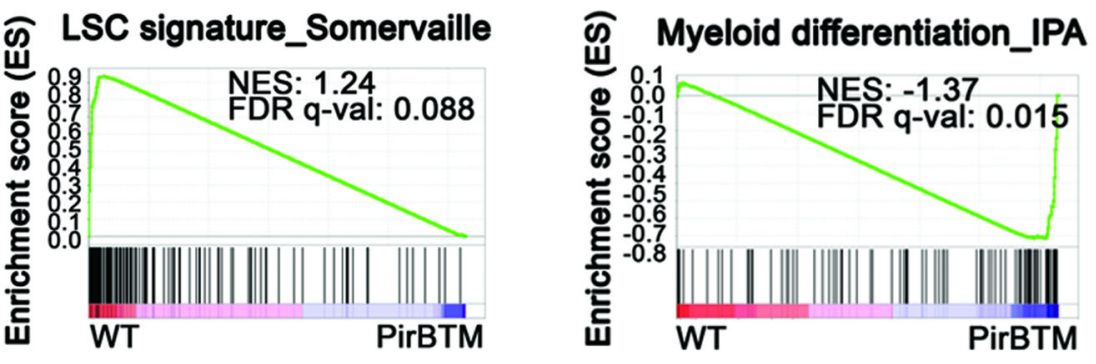

Figure 4. PirB suppresses differentiation and enhances development of MLL-AF9 AML

a, PirB expression on $\mathrm{YFP}^{+} \mathrm{Mac}-1^{+} \mathrm{Kit}^{+} \mathrm{AML}$ cells as determined by flow cytometery. $\mathbf{b}$, Survival curve of mice receiving MLL-AF9-infected WT or PirBTM hematopoietic progenitors $(n=15) ; \mathrm{p}<0.05$. c, Comparison of the sizes of spleen, liver, and numbers of peripheral blood cells of the mice transplanted with WT MLL-AF9 cells and those with PirBTM MLL-AF9 cells at 28 d after transplantation $(n=6)$. d, Representative flow cytometry plots showing that PirBTM AML mice have decreased Mac- $1^{+} \mathrm{Kit}^{+}$cells and increased differentiated cells relative to mice transplanted with WT cells at $28 \mathrm{~d}$ after transplantation. e, Comparison of colony forming activity of WT and PirBTM MLL-AF9+ BM cells. Shown is typical morphology of WT and PirBTM CFUs. f, PirBTM MLL-AF9 BM cells have dramatically decreased CFU forming ability in second replating $(n=3)$. g, GSEA plots evaluating changes in leukemia initiation/maintenance and myeloid 
differentiation gene signatures upon PirB signaling depletion in WT or PirBTM MLL-AF9 Mac- $1^{+} \mathrm{Kit}^{+}$AML cells. ${ }^{*} \mathrm{p}<0.05$. Error bars, s.e.m. 